$R M-75-61$

A CUTTING PLANE ALGORITHM FOR SOLVING BILINEAR PROGRAMS

Hiroshi Konno

December 1975

Research Memoranda are informal publications relating to ongoing or projected areas of research at IIASA. The views expressed are those of the author, and do not necessarily reflect those of IIASA. 


\section{A Cutting Plane Algorithm for Solving}

Bilinear Programs

Hiroshi Konno

\section{Introduction}

Nonconvex programs which have either a nonconvex minimand and/or a nonconvex feasible region have been considered by most mathematical programmers as a hopelessly difficult area of research. There are, however, two exceptions where considerable effort to obtain a global optimum is under way. One is integer linear programming and the other is nonconvex quadratic programming. This paper addresses itself to a special class of nonconvex quadratic program referred to as a 'bilinear program' in the literature. We will propose here a cutting plane algorithm to solve this class of problems. The algorithm is along the lines of [17] and [19] but the major difference is in its exploitation of special structure. Though the algorithm is not guaranteed at this stage to converge to a global optimum, the preliminary results are encouraging.

In section 2, we analyze the structure of the problem and develop an algorithm to obtain an $\varepsilon$-locally maximum pair of basic feasible solutions. In section 3 , we will generate a cutting plane to eliminate the current pair of $\varepsilon$-locally maximum basic feasible solutions. For these purposes, we extensively use the simplex algorithm. Section 4 gives an illustrative example and the results of numerical experimentations. Some of the important applications of bilinear programming can be found in references [11] and [12].

\section{Definitions and a Locally Maximum Pair of Basic Feasible} Solutions

The bilinear program is a class of quadratic programs with the following structure: 


$$
\begin{array}{r}
\max \phi\left(x_{1}, x_{2}\right)=c x_{1}^{t} x_{1}+c c_{2}^{t} x_{2}+x_{1}^{t} c x_{2} \\
\text { s.t. } A_{1} x_{1}=b_{1}, x_{1} \geq 0 \\
A_{2} x_{2}=b_{2}, x_{2} \geq 0,
\end{array}
$$

where $c_{i}, x_{i} \in R^{n_{i}}, b_{i} \varepsilon R^{m_{i}}, A_{i} \varepsilon R^{m_{i}^{\times n}} i, i=1,2$ and $c \varepsilon R^{n_{1} \times n_{2}} 2$. We will call this a bilinear program in 'standard' form.

Note that a bilinear program is a direct extension of the standard linear program: $\max \left\{c^{t} x \mid A x=b, x \geq 0\right\}$, in which we consider $c$ to be linearly constrained variables and maximize $c^{t} x$ with respect to $\mathrm{c}$ and $\mathrm{x}$ simultaneously. Let us denote

$$
x_{i}=\left\{\left.x_{i} \in R^{n}\right|_{i} x_{i}=b_{i}, x_{i} \geq 0\right\} \quad, \quad i=1,2 .
$$

Theorem 2.1. If $x_{i}, i=1,2$ are non-empty and bounded, then (2.1) has an optimal solution $\left(x_{1}^{*}, x_{2}^{*}\right)$ where $x_{i}^{*}$ is a basic feasible solution of the constraint equations defining $x_{i}, i=1,2$.

Proof. Let $\left(\hat{x}_{1}, \hat{x}_{2}\right)$ be an optimal solution, which clearly exists by assumption. Consider a linear program: $\max \left\{\phi\left(x_{1}, \hat{x}_{2}\right) \mid x_{1} \varepsilon x_{1}\right\}$, and let $x_{1}^{*}$ be its optimal basic solution. Then $\phi\left(\mathrm{x}_{1}^{*}, \hat{\mathrm{x}}_{2}\right) \geq \phi\left(\hat{\mathrm{x}}_{1}, \hat{\mathrm{x}}_{2}\right)$ since $\hat{\mathrm{x}}_{1}$ is a feasible solution to the linear program considered above. Next, consider another linear program: $\max \left\{\phi\left(\mathrm{x}_{1}^{*}, \mathrm{x}_{2}\right) \mid \mathrm{x}_{2} \in \mathrm{x}_{2}\right\}$, and let $\mathrm{x}_{2}^{*}$ be its optimal basic solution. Then by similar arguments as before, we have $\phi\left(x_{1}^{*}, x_{2}^{*}\right)$ $\geq \phi\left(\mathrm{x}_{1}^{*}, \hat{\mathrm{x}}_{2}\right)$. Thus we conclude that $\phi\left(\mathrm{x}_{1}^{*}, \mathrm{x}_{2}^{*}\right) \geq \phi\left(\hat{\mathrm{x}}_{1}, \hat{\mathrm{x}}_{2}\right)$, which implies that $\left(x_{1}^{*}, x_{2}^{*}\right)$ is a basic optimal solution of $(2.1)$.

Given a feasible basis $B_{i}$ of $A_{i}$, we will partition $A_{i}$ as $\left(B_{i}, N_{i}\right)$ assuming, without loss of generality, that the first $m_{i}$ columns of $A_{i}$ are basic. Position $x_{i}$ correspondingly: $x_{i}=\left(x_{i B}, x_{i N}\right)$. Let us introduce here a 'canonical' representation of (2.1) relative to a pair of feasible bases $\left(B_{1}, B_{2}\right)$. Premultiplying $B_{i}^{-1}$ to the constraint equation $B_{i} x_{i B}+N_{i N} x_{i N}=b_{i}$ and suppressing the basic variables $\mathbf{x}_{i B^{\prime}}$ we get the following 
$-3-$

system which is totally equivalent to (2.1):

$$
\begin{gathered}
\max \bar{\phi}\left(\mathrm{x}_{1 \mathrm{~N}}, \mathrm{x}_{2 \mathrm{~N}}\right)=\bar{c}_{1 \mathrm{~N}}^{-t} \mathrm{x}_{1 \mathrm{~N}}+\bar{c}_{2 \mathrm{~N}}^{-t} \mathrm{x}_{2 \mathrm{~N}}+\mathrm{x}_{1 \mathrm{~N}}^{\mathrm{t}} \overline{\mathrm{C}} \mathrm{x}_{2 \mathrm{~N}}+\phi\left(\mathrm{x}_{1}^{\circ}, \mathrm{x}_{2}^{\circ}\right) \\
\text { st. } \mathrm{B}_{1}^{-1} \mathrm{~N}_{1} \mathrm{x}_{1 \mathrm{~N}} \leq \mathrm{B}_{1}^{-1} \mathrm{~b}_{1}, \mathrm{x}_{1 \mathrm{~N}} \geq 0 \\
\mathrm{~B}_{2}^{-1} \mathrm{~N}_{2} \mathrm{x}_{2 \mathrm{~N}} \leq \mathrm{B}_{2}^{-1} \mathrm{~b}_{2}, \mathrm{x}_{2 \mathrm{~N}} \geq 0
\end{gathered}
$$

where

$$
x_{i}^{o} \equiv\left(x_{i B}^{0}, x_{i N}^{o}\right)=\left(B_{i}^{-1} b_{i}, 0\right)
$$

For future reference, we will introduce the notations

$$
\begin{aligned}
& \ell_{i}=n_{i}-m_{i}, d_{i}=\bar{c}_{i N} \varepsilon R^{\ell} i, y_{i}=x_{i N} \varepsilon R^{\ell}, \\
& F_{i}=B_{i}^{-1} N_{i} \varepsilon R^{m_{i} \times l} i \quad, \quad f_{i}=B_{i}^{-1} b_{i} \varepsilon R^{m_{i}}, i=1,2 \\
& Q=\bar{C} \varepsilon R^{\ell, l_{2}}, \phi_{0}=\phi\left(x_{1}^{\circ}, x_{2}^{\circ}\right)
\end{aligned}
$$

and rewrite $(2.3)$ as follows:

$$
\begin{array}{r}
\max \psi\left(y_{1}, y_{2}\right)=d_{1}^{t} y_{1}+d_{2}^{t} y_{2}+y_{1}^{t} Q y_{2} \\
\text { s.t. } F_{1} y_{1} \leq f_{1}, y_{1} \geq 0 \\
F_{2} y_{2} \leq f_{2}, y_{2} \geq 0 .
\end{array}
$$

We will call (2.4) a canonical representation of (2.1) relative to $\left(B_{1}, B_{2}\right)$ and use standard form (2.1) and canonical form (2.4) interchangeably, whichever is the more convenient for our presenration. To express the dependence of vectors in (2.4) on the pair of feasible bases $\left(B_{1}, B_{2}\right)$, we will occasionally use the notaction $d_{1}\left(B_{1}, B_{2}\right)$, etc. 
Theorem 2.2. The origin $\left(y_{1}, y_{2}\right)=(0,0)$ of the canonical system $(2.4)$ is

(i) a Kuhn-Tucker point if $d_{i} \leq 0$, $i=1,2$;

(ii) a local maximum if (a) and (b) hold:
(a) $a_{i} \leq 0, i=1,2$
(b) either $a_{1 i}<0$ or $a_{2 j}<0$ if $q_{i j}<0$;

(iii) a global optimum $a_{i} \leq 0, i=1,2$ and $Q \leq 0$.

\section{Proof.}

(i) It is straightforward to see that $y_{1}=0, y_{2}=0$ together with dual variables $u_{1}=0, u_{2}=0$ satisfy the KuhnTucker condition for (2.1).

(ii) Let $y_{i} \in R^{l}{ }^{l}, i=1,2$ be arbitrary nonnegative vectors. Let $J_{i}=\left\{j \mid q_{i j}<0\right\}$ and let $\varepsilon$ be positive scalar. Then

$$
\begin{aligned}
\psi\left(\varepsilon y_{1}, \varepsilon y_{2}\right) & =\varepsilon d_{1}^{t} y_{1}+\varepsilon d_{2}^{t} Y_{2}+\varepsilon y_{1}^{t} Q y_{2}+\phi_{o} \\
& \leqq \varepsilon \sum_{j \varepsilon J_{1}} d_{i j} Y_{i j}+\varepsilon \sum_{j \varepsilon J_{2}}^{\sum_{j}} d_{2 j} Y_{2 j}+\varepsilon^{2} \sum_{i \varepsilon J_{1}}^{\sum_{j \varepsilon J_{2}}}
\end{aligned}
$$

or

$$
q_{i j} Y_{1 i} y_{2 j}+\phi_{o}
$$

because $q_{i j} \leq 0$ when $i \not J_{1}$ and $j \not J_{2}$. Obviously, the last expression is equal to $\phi_{0}$ if $J_{1}=\phi$ and $J_{2}=\phi$. It is less than $\phi_{0}$ for $\operatorname{small}$ enough $\varepsilon$ if $J_{1} \neq \phi$ or $J_{2} \neq \phi$ since the linear term in $\varepsilon$ dominates the quadratic term. This implies that

$\psi\left(\varepsilon y_{1}, \varepsilon y_{2}\right) \leq \phi_{0}=\psi(0,0)$ for all $y_{1} \geq 0, y_{2} \geq 0$ and small enough $\varepsilon>0$.

(iii) This is obviously true since $\psi\left(y_{1}, y_{2}\right) \leq \phi_{0}=\psi(0,0)$ for all $y_{1} \geq 0, y_{2} \geq 0$.

The proof of Theorem 1 suggests to us a vertex following algorithm to be described below: 


\section{Algorithm 1 (Mountain Climbing)}

Step 1. Obtain a pair of basic feasible solutions, $\mathrm{x}_{1}^{\circ} \varepsilon \overline{\mathrm{x}_{1}, \mathrm{x}_{2}^{\circ}} \varepsilon \mathrm{x}_{2}$. Let $\mathrm{k}=0$.

Step 2. Given $\left(x_{1}^{k}, x_{2}^{k}\right)$, a pair of basic feasible solutions of $x_{1}$ and $x_{2}$, solve a subproblem: $\max \left\{\phi\left(x_{1}, x_{2}^{k}\right) \mid x_{1} \varepsilon x_{1}\right\}$. Let $\mathrm{x}_{1}^{\mathrm{k}+1}$ and $\mathrm{B}_{1}^{\mathrm{k}+1}$ be its optimal basic solution and corresponding basis.

Step 3. Solve a subproblem: $\max \left\{\phi\left(\mathrm{x}_{1}^{\mathrm{k}+1}, \mathrm{x}_{2}\right) \mid \mathrm{x}_{2} \varepsilon \mathrm{x}_{2}\right\}$, and let $\mathrm{x}_{2}^{\mathrm{k}+1}$ and $\mathrm{B}_{2}^{\mathrm{k}+1}$ be its optimal basic solution and corresponding basis.

Step 4. Compute $a_{1}\left(B_{1}^{k+1}, B_{2}^{k+1}\right)$, the coefficients of $y_{1}$ in the canonical representation $(2.4)$ relative to bases $\mathrm{B}_{1}^{\mathrm{k}+1}, \mathrm{~B}_{2}^{\mathrm{k}+1}$. If $a_{1}\left(B_{1}^{k+1}, B_{2}^{k+1}\right) \leq 0$, then let $B_{i}^{*}=B_{i}^{k+1}$ and $x_{i}^{*}$ be the basic feasible solutions associated with $B_{i}^{*}, i=1,2$ and HALT. Otherwise increase $\mathrm{k}$ by 1 and go to step 2 .

Note that the subproblems to be solved in Steps 2 and 3 are linear programs.

Proposition 2.3. If $x_{1}$ and $x_{2}$ are bounded, then Algorithm 1 halts in finitely many steps generating a Kuhn-Tucker point.

Proof. If every basis of $x_{1}$ is nondegenerate, then the value of objective function $\phi$ can be increased in step 2 as long as there is a positive component in $\mathrm{d}_{1}$. Since the number of bases of $x_{1}$ is finite and no pair of bases can be visited twice because the objective function is strictly increasing in each passage of step 2, the algorithm will eventually terminate with the condition $d_{1}\left(B_{1}^{k+1}, B_{2}^{k+1}\right) \leq 0$ being satisfied. When $x_{1}$ is degenerate, then there is a chance of infinite cycling among certain pairs of basic solutions. We will show, however, that this cannot happen in the above process if we employ an appropriate tie breaking device in linear programming. Suppose that 


$$
\begin{aligned}
& \phi\left(\mathrm{x}_{1}^{\mathrm{k}+1}, \mathrm{x}_{2}^{\mathrm{k}}\right)=\max \left\{\phi\left(\mathrm{x}_{1}, \mathrm{x}_{2}^{\mathrm{k}}\right) \mid \mathrm{x}_{1} \varepsilon \mathrm{x}_{1}\right\} \quad \text { optimal basis } \\
& \phi\left(\mathrm{x}_{1}^{\mathrm{k}+1}, \mathrm{x}_{2}^{\mathrm{k}+1}\right)=\max \left\{\phi\left(\mathrm{x}_{1}^{\mathrm{k}+1}, \mathrm{x}_{2}\right) \mid \mathrm{x}_{2} \varepsilon \mathrm{x}_{2}\right\} \quad: \mathrm{B}_{2}^{\mathrm{k}+1} \\
& \cdot \cdot \cdot \cdot \cdot \cdot \\
& \cdot \cdot \cdot \cdot \cdot \cdot \\
& \phi\left(\mathrm{x}_{1}^{\mathrm{k}+\ell}, \mathrm{x}_{2}^{\mathrm{k}+\ell-1}\right)=\max \left\{\phi\left(\mathrm{x}_{1}, \mathrm{x}_{2}^{\mathrm{k}+\ell-1}\right) \mid \mathrm{x}_{1} \varepsilon \mathrm{x}_{1}\right\}: \mathrm{B}_{1}^{\mathrm{k}+\ell} \\
& \phi\left(\mathrm{x}_{1}^{\mathrm{k}+\ell}, \mathrm{x}_{2}^{\mathrm{k}+\ell}\right)=\max \left\{\phi\left(\mathrm{x}_{1}^{\mathrm{k}+\ell}, \mathrm{x}_{2}\right) \mid \mathrm{x}_{2} \varepsilon \mathrm{x}_{2}\right\} \quad: \mathrm{B}_{2}^{\mathrm{k}+\ell},
\end{aligned}
$$

where $x^{k+l}=x^{k+1}$, for the first time in the cycle. Since the value of objective function $\phi$ is nondecreasing and

$$
\left(x_{1}^{k+\ell}, x_{2}^{k+\ell}\right) \equiv \phi\left(x_{1}^{k+1}, x_{2}^{k+l}\right) \leq \phi\left(x_{1}^{k+1}, x_{2}^{k+1}\right)
$$

we have that

$$
\phi\left(x_{1}^{k+1}, x_{2}^{k+1}\right)=\phi\left(x_{1}^{k+2}, x_{2}^{k+1}\right)=\ldots .=\phi\left(x_{1}^{k+l}, x_{2}^{k+l}\right)
$$

It is obvious that $\mathrm{d}_{2}\left(\mathrm{~B}_{1}^{\mathrm{k}+1}, \mathrm{~B}_{2}^{\mathrm{k}+1}\right) \leq 0$ by the definition of optimality of $B_{2}^{k+1}$. Suppose that the $j$ th component of $d_{1}\left(B_{1}^{k+1}, B_{2}^{k+1}\right)$ is positive. Then we could have introduced $y_{i j}$ into the basis. However, since the objective function should not increase, $y_{i j}$ comes into the basis at zero level. Hence the vector $y_{1}$ remains zero. We can eliminate the positive element of $\mathrm{d}_{1}$, one by one (using tie breaking device for the degenerate LP if necessary) with no actual change in the value of $y_{1}$. Eventually, we have $\mathrm{d}_{2} \leq 0$ with $\mathrm{Y}_{1}=0$ and the corresponding basis $\tilde{\mathrm{B}}_{1}^{\mathrm{k}+1}$. Referring to the standard form, the corresponding $x_{1}$ value remains unchanged i.e., stays at $x_{1}^{k+1}$ and hence $d_{2}\left(\tilde{B}_{1}^{k+1}, B_{2}^{k+1}\right) \leq 0$, because $\mathrm{B}_{2}^{\mathrm{k}+1}$ is the optimal basis for $\mathrm{x}_{1}=\mathrm{x}_{1}^{\mathrm{k}+1}$, and $\tilde{\mathrm{x}}_{1}^{\mathrm{k}+1}=\mathrm{x}_{1}^{\mathrm{k}+1}$. By Theorem 2 (i), the solution obtained is a Kuhn-Tucker point. 
Let us assume in the following that a Kuhn-Tucker point has been obtained and that a canonical representation (2.4) relative to the associated pair of bases has been given.

By Theorem 2 (iii), that pair of basic feasible solutions is optimal if $Q \leq 0$. We will assume that this is not the case and let

$$
K=\left\{(i, j) \mid q_{i j}>0\right\} .
$$

Let us define for $(i, j) \in K$, a function $\psi_{i j}: R_{+}^{2} \rightarrow R_{\text {, }}$

$$
\psi_{i j}(\xi, n)=d_{1 i} \xi+d_{2 j} n+q_{i j} \xi \eta
$$

Proposition 2.4. If $\psi_{i j}\left(\xi_{0}, \eta_{0}\right)>0$ for some $\xi_{0} \geq 0, \eta_{0} \geq 0$, then

$$
\psi_{i j}(\xi, \eta)>\psi\left(\xi_{0}, \eta_{0}\right) \text { for all } \xi>\xi_{0}, \eta>\eta_{0}
$$

Proof.

$$
\begin{aligned}
& \psi_{i j}(\xi, n)-\psi_{i j}\left(\xi_{0}, n_{0}\right)=\left(\xi-\xi_{0}\right)\left(d_{1 i}+q_{i j} n_{0}\right) \\
& +\left(n-\eta_{0}\right)\left(d_{2 j}+q_{i j} \xi_{0}\right) \\
& +q_{i j}\left(\xi-\xi_{0}\right)\left(n-\eta_{0}\right) \\
& \geq\left(\xi-\xi_{0}\right)\left(-d_{2 j} \frac{n_{o}}{\xi_{0}}\right) \\
& +\left(\eta-\eta_{0}\right)\left(-d_{1 i} \frac{\xi_{0}}{\eta_{0}}\right) \\
& +q_{i j}\left(\xi-\xi_{0}\right)\left(\eta-\eta_{0}\right)>0 . \quad \mid 1
\end{aligned}
$$

This proposition states that if the objective function increases in the directions of $y_{1 j}$ and $y_{2 j}$, then we can increase more if we go further into this direction. 
Definition 2.1. Given a basic feasible solution $x_{i} \varepsilon x_{i}$, let $\mathrm{N}_{i}\left(\mathrm{x}_{i}\right)$ be the set of adjacent basic feasible solutions which can be reached from $x_{i}$ in one pivot step.

Definition 2.2. Let $\varepsilon$ be a nonnegative scalar. A pair of basic feasible solutions $\left(x_{1}^{*}, x_{2}^{*}\right), x_{i}^{*} \varepsilon x_{i}, i=1,2$ is called an $\varepsilon$-locally maximum pair of basic feasible solution if

$$
d_{i} \leq 0, i=1,2
$$

$$
\phi\left(\mathrm{x}_{1}^{*}, \mathrm{x}_{2}^{*}\right) \geq \phi\left(\mathrm{x}_{1}, \mathrm{x}_{2}\right)-\varepsilon \text { for all } \mathrm{x}_{i} \varepsilon \mathrm{N}_{i}\left(\mathrm{x}_{i}^{*}\right), i=1,2 \text {. }
$$

Given a Kuhn-Tucker point $\left(\mathrm{x}_{1}^{*}, \mathrm{x}_{2}^{*}\right)$, we will compute $\phi\left(\mathrm{x}_{1}, \mathrm{x}_{2}\right)$ for all $x_{i} \varepsilon N_{i}\left(x_{i}^{*}\right), i=1,2$ for which a potential increase of objective function $\phi$ is possible. Given a canonical representation, it is sufficient for this purpose to calculate $\psi_{i j}\left(\bar{\xi}_{i}, \bar{n}_{j}\right)$ for $(i, j) \varepsilon k$ where $\bar{\xi}_{i}$ and $\bar{n}_{j}$ represent the maximum level of nonbasic variables $x_{1 j}$ and $x_{2 j}$ when they are introduced into the bases without violating feasibility.

\section{Algorithm 2 (Augmented Mountain Climbing)}

Step 1. Apply Algorithm 1 and let $x_{i}^{*} \varepsilon x_{i}, i=1,2$ be the resulting pair of basic feasible solutions.

Step 2. If $\left(x_{1}^{*}, x_{2}^{*}\right)$ is an $\varepsilon-1 o c a l l y$ maximum pair of basic feasible solutions, then HALT. Otherwise, move to the adjacent pair of basic feasible solutions $\left(\hat{x}_{1}, \hat{x}_{2}\right)$ where

$$
\phi\left(\hat{x}_{1}, \hat{x}_{2}\right)=\max \left\{\phi\left(\mathrm{x}_{1}, \mathrm{x}_{2}\right) \mid \mathrm{x}_{i} \in \mathrm{N}_{i}\left(\mathrm{x}_{i}^{*}\right), \quad i=1,2\right\}
$$

and go to step 1 .

Proposition 2.5. If $\mathrm{x}_{1}$ and $\mathrm{x}_{2}$ are bounded and if $\varepsilon>0$, Algorithm 2 halts in finitely many steps generating an $\varepsilon$-locally maximum pair of basic feasible solutions.

Proof. It follows immediately from the following facts that: 
(i) step 1 converges in finitely many steps (by Proposition 2.3),

(ii) whenever we pass step 2, the value of the objective function is improved by at least $\varepsilon(>0)$,

(iii) there are only finitely many basic feasible solutions for $\mathrm{x}_{1}$ and $\mathrm{x}_{2}$.

\section{Cutting Planes}

We will assume in this section that an $\varepsilon$-locally maximum pair of basic feasible solutions has been obtained and that a canonical representation relative to this pair of basic feasible solution $\left(x_{1}^{*}, x_{2}^{*}\right)$ has been given. Since we will refer here exclusively to a canonical representation, we will reproduce it for future convenience:

$$
\begin{gathered}
\max \psi\left(y_{1}, y_{2}\right)=d_{1}^{t} y_{1}+d_{2}^{t} y_{2}+y_{1}^{t} Q y_{2}+\phi\left(x_{1}^{*}, x_{2}^{*}\right) \\
\text { s.t. } F_{1} y_{2} \leq f_{1}, y_{1} \geq 0 \\
F_{2} y_{2} \leq E_{2}, y_{2} \geq 0,
\end{gathered}
$$

where

$$
\mathrm{d}_{i} \leq 0, \mathrm{f}_{\mathrm{i}} \geq 0, \mathrm{i}=1,2 .
$$

Let

$$
\begin{aligned}
Y_{i}=\left\{y_{i} \varepsilon R^{l} \mid F_{i} y_{i} \leq E_{i}, y_{i} \geq 0\right\}, i=1,2 \\
Y_{i}^{(l)}=\left\{\left.y_{i} \varepsilon R^{l}\right|_{y_{i l}} \geq 0, y_{i j}=0, j \neq l\right\} \\
\ell=1, \ldots, l_{i}, i=1,2,
\end{aligned}
$$

i.e. $Y_{i}^{(\ell)}$ is the ray emanating from $y_{i}=0$ in the direction $y_{i \ell}$. 
Lemma 3.1. Let

$$
\psi_{1}(\cdot)=\max \left\{\psi\left(\cdot, y_{2}\right) \mid y_{2} \in y_{2}\right\}
$$

If $\Psi_{1}(u)>0$ for some $u \varepsilon Y_{1}^{(l)}$, then $\Psi_{1}(v)>\Psi_{1}(u)$ for all $\mathrm{V} \varepsilon \mathrm{Y}_{1}^{(\ell)}$ such that $\mathrm{v}>\mathrm{u}$.

Proof. Let $u=\left(0, \ldots, 0, u_{\ell}, 0, \ldots, 0\right)$. First note that $u_{\ell}>0$, since if $u_{\ell}=0$, then $\Psi_{1}(u)=\max \left\{d_{2}^{t} y_{2} \mid y_{2} \varepsilon y_{2}\right\}=0$.

Let $v=\left(0, \ldots, 0, v_{\ell}, 0, \ldots, 0\right)$ where $v_{\ell} \geq u_{\ell}$. Then for all $\mathrm{Y}_{2} \in \mathrm{Y}_{2}$, we have

$$
\begin{aligned}
\psi\left(v_{,} y_{2}\right) & =\psi\left(u, y_{2}\right)+\left(v_{\ell}-u_{\ell}\right)\left\{d_{1 \ell}+\sum_{j=1}^{\ell} q_{\ell j} y_{2 j}\right\} \\
& \geq \psi\left(u, y_{2}\right)+\frac{v_{\ell}-u_{\ell}}{u_{\ell}}\left\{d_{1 \ell} u_{\ell}+\sum_{j=1}^{\ell}\left(d_{2 j}+q_{\ell j} u_{\ell}\right) y_{2 j}\right\} \\
& =\frac{v_{\ell}}{u_{\ell}} \psi\left(u, y_{2}\right) .
\end{aligned}
$$

The inequality follows from $d_{2} \leq 0$. Thus

$$
\begin{aligned}
\max \left\{\psi\left(\mathrm{v}, \mathrm{Y}_{2}\right) \mid \mathrm{Y}_{2} \in \mathrm{Y}_{2}\right\} & \geq \frac{\mathrm{v}_{\ell}}{\mathrm{u}_{\ell}} \max \left\{\psi\left(\mathrm{u}, \mathrm{Y}_{2}\right) \mid \mathrm{Y}_{2} \varepsilon \mathrm{Y}_{2}\right\} \\
& \geq \max \left\{\psi\left(\mathrm{u}, \mathrm{Y}_{2}\right) \mid \mathrm{Y}_{2} \varepsilon \mathrm{Y}_{2}\right\} .
\end{aligned}
$$

This lemma shows that the function $\Psi_{1}$ is a strictly increasing function of $\mathrm{Y}_{1}$ on $\mathrm{Y}_{1}^{(l)}$ beyond the point where $\psi_{1}$ first becomes positive. 


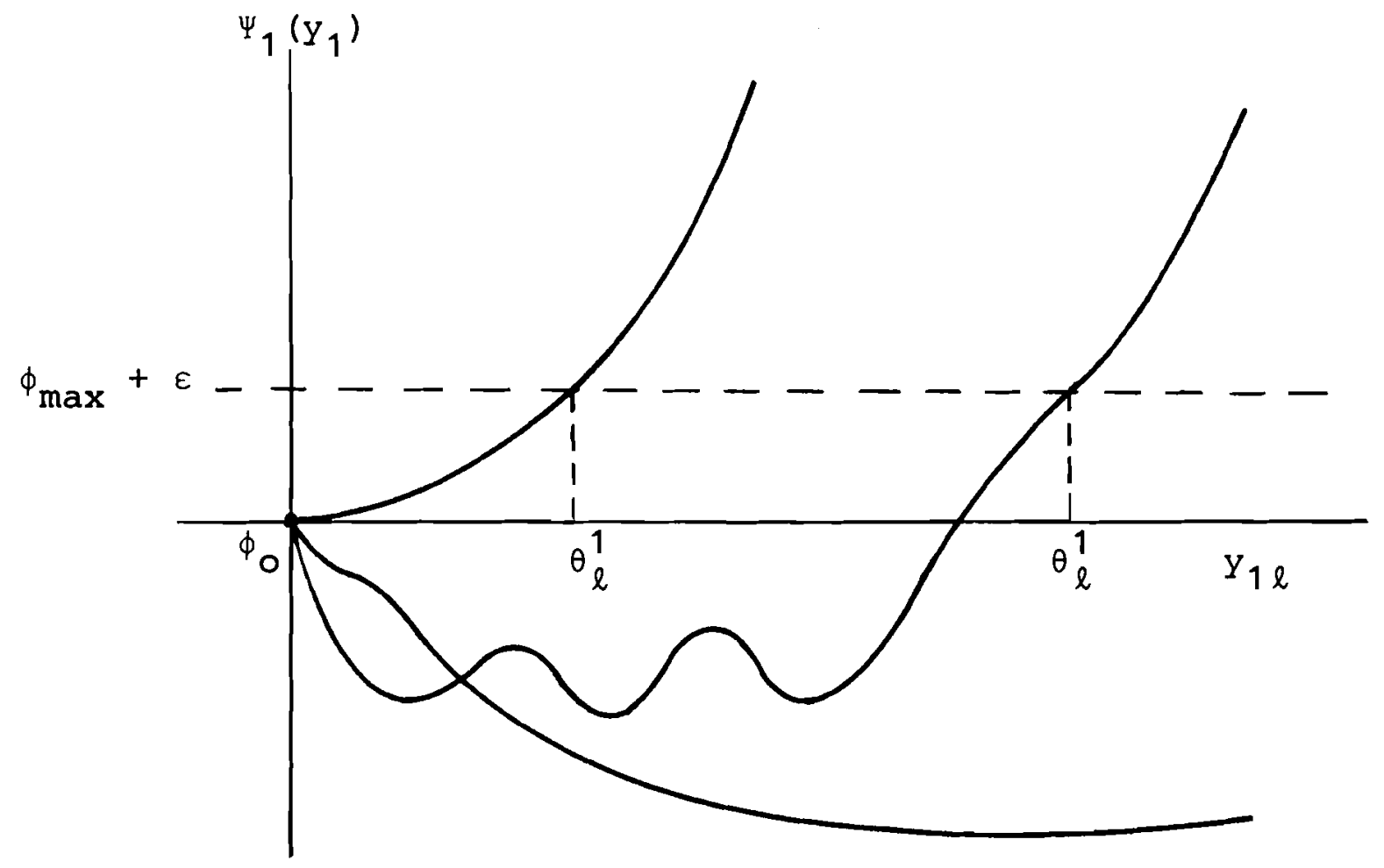

Figure 3.1. Shape of the function $\Psi_{1}$.

Let $\phi_{\max }$ be the value of the objective function associated with the best feasible solution obtained so far by one method or another and let us define $\theta_{l}^{1}, \ell=1, \ldots, \ell_{1}$ as follows:

$$
\begin{aligned}
& \theta_{\ell}^{1}=\max \theta \text { for which } \\
& \max \left\{\Psi_{1}\left(y_{1}\right) \mid Y_{1} \varepsilon Y_{1}^{(\ell)}, 0 \leq y_{1 \ell} \leq \theta\right\} \leq \phi_{\max }+\varepsilon
\end{aligned}
$$

Lemma 3.2. $\theta_{\ell}^{1}>0, \ell=1, \ldots, \ell_{1}$.

Proof. Let $y_{1}=\left(0, \ldots, 0, y_{1 \ell}, 0, \ldots, 0\right)$. since $d_{1} \leq 0$, $\mathrm{d}_{2} \leq 0$, we have

$$
\begin{aligned}
\Psi\left(y_{1}, y_{2}\right) & =d_{1 \ell} y_{1 \ell}+\sum d_{2 j} y_{2 j}+y_{1 \ell} \sum q_{\ell j} y_{2 j}+\phi_{o} \\
& \leq y_{1 \ell} \sum q_{\ell j} y_{2 j}+\phi_{0} .
\end{aligned}
$$


Letting $\alpha=\max \left\{\Sigma q{ }_{j} Y_{2 j} Y_{2} \quad \varepsilon Y_{2} \geq 0\right.$, we know from the above inequality that

$$
\theta_{\ell}^{1} \begin{cases}\geq\left(\phi_{\max }-\phi_{O}+\varepsilon\right) / \alpha>0 & \alpha>0 \\ =+\infty & \alpha=0 .\end{cases}
$$

Theorem 3.3. Let

$$
\Delta_{1}\left(\theta^{1}\right)=\left\{y_{1} \varepsilon R^{\ell_{1}} \sum_{j=1}^{\ell_{1}} y_{1 j} / \theta_{j}^{1} \leq 1, y_{1} \geq 0\right\}
$$

Then

$\max \left\{\psi\left(y_{1}, y_{2}\right) \mid y_{1} \varepsilon \Delta_{1}\left(\theta^{1}\right), y_{2} \varepsilon Y_{2}\right\} \leq \phi_{\max }+\varepsilon$.

Proof. Let

$$
\tilde{\theta}_{j}^{1}= \begin{cases}\theta_{j}^{1} & \text { if } \theta_{j}^{1} \text { is finite } \\ \theta_{0} & \text { if } \theta_{j}^{1}=\infty\end{cases}
$$

where $\theta_{0}>0$ is constant. Then

$$
\begin{aligned}
& \max \left\{\psi\left(y_{1}, y_{2}\right) \mid y_{1} \varepsilon \Delta_{1}\left(\theta^{1}\right), y_{2} \varepsilon y_{2}\right\} . \\
& =\lim _{\substack{\rightarrow \infty}} \max \left\{\psi\left(y_{1}, y_{2}\right) \mid y_{1} \varepsilon \Delta_{1}\left(\tilde{\theta}^{1}\right), y_{2} \varepsilon y_{2}\right\} .
\end{aligned}
$$

The right hand term inside the limit is a bilinear program with bounded feasible region, and hence by Theorem 2.1, there exists an optimal solution among basic feasible solutions. Since the basic feasible solution for the systems of inequalities defining $\Delta\left(\tilde{\theta}^{1}\right)$ are $(0, \ldots, 0)$ and $y_{1}^{\ell}=\left(0, \ldots, 0, \tilde{\theta}_{\ell}^{1}, 0, \ldots, 0\right), \ell=1, \ldots, \ell_{1}$, we have 
$\begin{aligned} \max & \left\{\psi\left(\mathrm{Y}_{1}, \mathrm{y}_{2}\right) \mid \mathrm{y}_{1} \in \Delta_{1}\left(\tilde{\theta}_{1}\right), \mathrm{y}_{2} \in \mathrm{Y}_{2}\right\} \\ & =\max \left[\max \left\{\psi\left(0, \mathrm{y}_{2}\right) \mid \mathrm{Y}_{2} \in \mathrm{Y}_{2}\right\}, \max _{\ell} \max _{\mathrm{y}_{2}}\left\{\psi\left(\mathrm{y}_{1}^{\ell}, \mathrm{y}_{2}\right) \mid \mathrm{y}_{2} \in \mathrm{Y}_{2}\right\}\right] .\end{aligned}$

However, since $d_{2} \leq 0$,

$\max \left\{\psi\left(0, y_{2}\right) \mid y_{2} \varepsilon y_{2}\right\}=\max \left\{d_{2}^{t} y_{2} \mid y_{2} \varepsilon y_{2}\right\}+\phi_{0} \leq \phi_{0} \leq \phi_{\max }+\varepsilon$.

Also,

$$
\max _{y_{2}}\left\{\psi\left(y_{1}, y_{2}\right) \mid y_{2} \in y_{2}\right\} \leq \phi_{\max }+\varepsilon
$$

by the definition of $\tilde{\theta}_{\ell}^{1}($ See $(3.5)$ and $(3.7))$. Hence

$$
\lim _{\substack{0 \\ \theta_{\infty}}} \max \left\{\psi\left(y_{1}^{\ell}, y_{2}\right) \mid y_{2} \varepsilon y_{2}\right\} \leq \phi_{\max }+\varepsilon
$$

This theorem shows that the value of the objective function $\phi\left(y_{1}, y_{2}\right)$ associated with the points $y_{1}$ in the region $Y_{1} \cap \Delta_{1}\left(\theta^{1}\right)$ is not greater than $\phi_{\max }+\varepsilon$ regardless of the choice of $\mathrm{Y}_{2} \varepsilon \mathrm{Y}_{2}$ and hence this region $Y_{1} \cap \Delta_{1}\left(\theta^{1}\right)$ can be ignored in the succeeding process to obtain an $\varepsilon$-optimal solution. The cut

$$
H_{1}\left(\theta^{1}\right): \sum_{j=1}^{\ell} Y_{1 j} / \theta_{j}^{1} \geq 1
$$

is, therefore, a 'valid' cut in the sense that it:

(i) does not contain the current $\varepsilon-l o c a l l y$ maximum pair of basic feasible solutions;

(ii) contains all the candidates $y_{1} \varepsilon Y_{1}$ for which

$$
\max \left\{\psi\left(y_{1}, y_{2}\right) \mid y_{2} \varepsilon Y_{2}\right\}>\phi_{\max }+\varepsilon
$$


Since $\theta^{1}$ is dependent on the feasible region $Y_{2}$, we will occasionally use the notation $\theta^{1}\left(\mathrm{Y}_{2}\right)$.

Since the problem is symmetric with respect to $\mathrm{Y}_{1}$ and $\mathrm{Y}_{2}$ ' we can, if we like, interchange the role of $\mathrm{Y}_{1}$ and $\mathrm{Y}_{2}$ to obtain another valid cutting plane relative to $\mathrm{Y}_{2}$ :

$$
H_{2}\left(\theta^{2}\right): \sum_{j=1}^{\ell} Y_{2 j} / \theta_{j}^{2}=1 \text {. }
$$

\section{Cutting Plane Algorithm}

Step 0. Set $\ell=0$. Let $x_{i}^{0}=x_{i}, i=1,2$.

Step 1. Apply Algorithm 2 (Augmented Mountain Climbing Algorithm) with a pair of feasible regions $x_{1}^{\ell}, x_{2}^{l}$.

Step 2. Compute $\theta^{1}\left(\mathrm{Y}_{2}^{\ell}\right)$. Let $\mathrm{Y}_{1}^{\ell+1}=\mathrm{Y}_{1}^{\ell} \triangle_{1}\left(\theta^{1}\left(\mathrm{Y}_{2}^{\ell}\right)\right.$. If $\mathrm{Y}_{1}^{\ell+1}=\phi$, stop. Otherwise proceed to the next step. Step 2' (Optional). Compute $\theta^{2}\left(\mathrm{Y}_{1}^{\ell+1}\right)$. Let $\mathrm{Y}_{2}^{\ell+1}=\mathrm{Y}_{2} \backslash \Delta_{2}\left(\theta^{2}\left(\mathrm{Y}_{1}^{\ell+1}\right)\right)$. If $\mathrm{Y}_{2}^{\ell+1}=\phi$, stop. Otherwise proceed to the next step.

Step 3. Add 1 to $\ell$. Go to step 1 .

It is now easy to prove the following theorem.

Theorem 3.4. If the cutting plane algorithm defined above stops in Step 2 or $2^{\prime}$, with either $\mathrm{Y}_{1}^{\ell+1}$ or $\mathrm{Y}_{2}^{\ell+1}$ becoming empty, then $\phi_{\max }$ and the associated pair of basic feasible solutions is an $\varepsilon$-optimal solution of the bilinear program.

Proof. Each cutting plane added does not eliminate any point for which the objective function is greater than $\phi_{\max }+\varepsilon$. Hence if either $\mathrm{Y}_{1}^{\ell+1}$ or $\mathrm{Y}_{2}^{\ell+2}$ becomes empty, we can conclude that $\max \left\{\psi\left(\mathrm{Y}_{1}, \mathrm{Y}_{2}\right) \mid \mathrm{Y}_{1} \varepsilon \mathrm{Y}_{1}, \mathrm{Y}_{2} \varepsilon \mathrm{Y}_{2}\right\} \leq \phi_{\max }+\varepsilon$.

According to our cutting plane algorithm, the number of constraints increases by 1 whenever we pass step 2 or 2 ', the size of subproblem becomes bigger and the constraints are also more prone to degeneracy. From this viewpoint, we want to add 
a smaller number of cutting planes, particularly when the original constraints have a good structure. In such cases, we might as well omit step $2^{\prime}$, taking $\mathrm{Y}_{2}$ as the constraints throughout the whole process.

Another requirement for the cut is that it should be as deep as possible, in the following sense.

Definition 3.1. Let $\theta=\left(\theta_{j}\right)>0, \tau=\left(\tau_{j}\right)>0$. Then the cut $\sum \mathrm{y}_{1 j^{\prime}} \mathrm{\theta}_{j} \geq 1$ is deeper $\operatorname{than} \sum \mathrm{y}_{1 j^{\prime}} \tau_{j} \geq 1$ if $\theta \geq \tau$, with at least one component with strict inequality.

Looking back into the definition (3.5) of $\theta^{1}$, it is clear that $\theta^{1}(\mathrm{U}) \geq \theta^{1}(\mathrm{~V})$ when $\mathrm{U} \subset \mathrm{V} \subset \mathrm{R}^{l} 2$ and that the cut associated with ${ }^{1}(U)$ is deeper than $\theta^{1}(V)$. This observation leads to the following procedure.

Iterative Improvement Procedure. Let $\mathrm{H}_{1}\left(\theta^{1}\left(\mathrm{Y}_{2}\right)\right)$ and $\mathrm{H}_{2}\left(\theta^{2}\left(\mathrm{Y}_{1}\right)\right)$ be a pair of valid cuts and let $\mathrm{Y}_{1}^{\prime}=\mathrm{Y}_{1} \|_{1}\left(\theta^{\prime}\left(\mathrm{Y}_{2}\right)\right)$, $\mathrm{Y}_{2}^{\prime}=\mathrm{Y}_{2} \backslash \Delta_{2}\left(\theta^{2}\left(\mathrm{Y}_{1}\right)\right)$ be the shrunken feasible regions. Generate cuts $H_{1}\left(\theta^{1}\left(Y_{2}^{\prime}\right)\right)$ and $H_{2}\left(\theta^{2}\left(Y_{1}^{\prime}\right)\right)$ which are generally deeper than $\mathrm{H}_{1}\left(\theta^{1}\left(\mathrm{Y}_{2}\right)\right)$ and $\mathrm{H}_{2}\left(\theta^{2}\left(\mathrm{Y}_{1}\right)\right)$, respectively. Iterate this process until successive cuts converge within some tolerance.

This iterative improvement scheme is very powerful when the problem is symmetric with respect to $\mathrm{y}_{1}$ and $\mathrm{y}_{2}$ :

$\max \left\{d^{t} t_{1}+d^{t} y_{2}+y_{1}^{t} Q y_{2} \mid F y_{1} \leq f, y_{1} \geq 0, F y_{2} \leq f, y_{2} \geq 0\right\}$.

In particular, maximization of a convex quadratic function subject to linear constraints

$$
\max \left\{2 d^{t} x+x^{t} Q x \mid F x \leq f, x \geq 0\right\}
$$

is equivalent to (3.8) and the iterative process described above works remarkably well for this class of problems. The details about this, together with the comparison of our cuts with the ones proposed by Tui and Ritter, will be discussed in full in [11]. 
The following theorem gives us a method to compute $\theta^{1}$ using the dual simplex method.

Theorem 3.5.

$$
\begin{aligned}
\theta_{l}^{1}=\min \left\{-d^{t} z+\left(\phi_{\max }-\phi_{0}+\varepsilon\right) z_{0}\right\} \\
\text { s.t. } F_{2} z-f_{2} z_{0} \leq 0 \\
l_{j=1}^{\ell}{ }^{2}{ }_{l j}{ }^{j}+d_{1 \ell} z_{0}=1 \\
z_{j} \geq 0, j=1, \ldots, 2^{\prime}, z_{0} \geq 0 .
\end{aligned}
$$

Proof. Let

$$
\begin{aligned}
& g(\theta)=\max \left\{d_{1}^{t} y_{1}+d_{2}^{t} y_{2}+y_{1}^{t} Q y_{2} \mid F_{2} y_{2} \leq f_{2}, y_{2} \geq 0\right. \\
& \left.0 \leq y_{1 l} \leq \theta, y_{1 j}=0, j \neq l\right\} .
\end{aligned}
$$

$\theta_{\ell}$ is then given as the maximum of $\theta$ for which $g(\theta) \leq \phi_{\max }-\phi_{0}$ $+\varepsilon$. It is not difficult to observe that

$$
g(\theta)=\max \left[0, \max \left\{d_{1 \ell} \theta+\left(d_{2}+\theta q_{\ell} \cdot\right)^{t} y_{2} \mid F_{2} y_{2} \leq f_{2}, y_{2} \geq 0\right\}\right]
$$

where $q_{\ell \cdot}=\left(q_{\ell 1}, \ldots, q_{\ell \ell}\right)^{t}$. Therefore, $\theta_{\ell}^{1}$ is the maximum of $\theta$ for which

$$
\begin{array}{r}
g_{1}(\theta) \equiv \max \left\{d_{1 \ell} \theta+\left(d_{2}+\theta q_{\ell \cdot}\right)^{t} y_{2} \mid F_{2} y_{2} \leq f_{2}, y_{2} \geq 0\right\} \\
\leq \phi_{\max }-\phi_{0}+\varepsilon
\end{array}
$$


The feasible region defining $g_{1}(\theta)$ is, by assumption, bounded and non-empty, and by art duality theorem

$$
g_{1}(\theta)=\min \left\{f_{2}^{t} u+d_{1 \ell} \theta \mid F_{2}^{t} u \geq d_{2}+\theta q_{\ell}, u \geq 0\right\} .
$$

Hence $\theta_{\ell}$ is the maximum of $\theta$ for which the system

$$
\left\{\mathrm{f}_{2}^{\mathrm{t}} \mathrm{u}+\mathrm{d}_{1 \ell}{ }^{\theta} \leq \phi_{\max }-\phi_{0}+\varepsilon,-\mathrm{F}_{2}^{\mathrm{t}} \mathrm{u}-\mathrm{q}_{\ell \cdot \theta} \leq-\mathrm{d}_{2}, \mathrm{u} \geq 0\right\}
$$

is feasible, i.e.,

$$
\theta_{\ell}=\max \left\{\begin{array}{l}
\theta \mid \begin{array}{l}
\mathrm{f}_{2}^{t} \mathrm{u}+\mathrm{a}_{1 \ell^{\theta}} \leq \phi_{\max }-\phi_{0}+\varepsilon \\
-\mathrm{F}_{2}^{\mathrm{t}} \mathrm{u}-\mathrm{q}_{\ell} \theta \leq-\mathrm{a}_{2} \\
\mathrm{u} \geq 0
\end{array}
\end{array}\right\}
$$

This problem is always feasible, and again using art duality theorem,

$$
\theta_{l}=\min \left\{\begin{array}{l|l}
-d_{2}^{t} z+\left(\phi_{\max }-\phi_{0}+\varepsilon\right) z_{0} & \begin{array}{l}
q_{l}^{t} z+d_{1 \ell} z_{0}=1 \\
f_{2} z_{0}-F z \geq 0 \\
z \geq 0, z_{0} \geq 0
\end{array}
\end{array}\right\}
$$

with the usual understanding that $\theta_{l}=+\infty$ if the constraint set above is empty.

Note that $\mathrm{d}_{2} \leq 0$ and $\phi_{\max }-\phi_{0}+\varepsilon \geq 0$ and hence $\left(z, z_{0}\right)=(0,0)$ is a dual feasible solution. Also the linear program defining $\theta_{l}^{1}$ is only one row different for different $\ell$, so that they are expected to be solved without an excessive amount of computation. Since the value of the objective function of (3.9) approaches its minimal value monotonically from below, we can stop pivoting if we like when the value of the objective function becomes greater than some specified value. The 
important thing to note is that if we pivot more, we get a deeper cut, in general.

\section{Numerical Examples}

Let us consider the following simple two dimensional example (illustrated in Figure 4.1):

$$
\begin{gathered}
\text { maximize } \phi\left(x_{1}, x_{2}\right)=(-1,1)\left(\begin{array}{l}
x_{11} \\
x_{12}
\end{array}\right)+(1,0)\left(\begin{array}{l}
x_{21} \\
x_{22}
\end{array}\right) \\
+\left(x_{11}, x_{12}\right)\left(\begin{array}{rr}
1 & -1 \\
-1 & 1
\end{array}\right)\left(\begin{array}{l}
x_{21} \\
x_{22}
\end{array}\right) \\
\operatorname{s.t.}\left(\begin{array}{ll}
1 & 4 \\
4 & 1 \\
3 & 4
\end{array}\right)\left(\begin{array}{l}
x_{11} \\
x_{12}
\end{array}\right) \leq\left(\begin{array}{l}
8 \\
12 \\
12
\end{array}\right),\left(\begin{array}{ll}
2 & 1 \\
1 & 2 \\
1 & 1
\end{array}\right)\left(\begin{array}{l}
x_{21} \\
x_{22}
\end{array}\right) \leq\left(\begin{array}{l}
8 \\
8 \\
5
\end{array}\right) \\
\left(x_{11}, x_{12}\right) \geq 0, \\
\left(x_{21}, x_{22}\right) \geq 0
\end{gathered}
$$

There are two locally maximum pairs of basic feasible solutions i.e., $\left(P_{1}, Q_{1}\right)$ and $\left.P_{4}, Q_{4}\right)$, for which the value of the objective function is 10 and 13, respectively. We applied the algorithm omitting step 2'. Two cuts generated at $\mathrm{P}_{1}$ and $\mathrm{P}_{4}$ are shown on the graph. In two steps, $x_{1}^{2}=\phi$ and the global optimum $\left(P_{4}, Q_{4}\right)$ has been identified.

We have coded the algorithm in FORTRAN IV for CYBER 74 at the Technische Hochschule, Vienna, and tested it for various problems of a size up to $10 \times 22,13 \times 24$; all of them were solved successfully. 

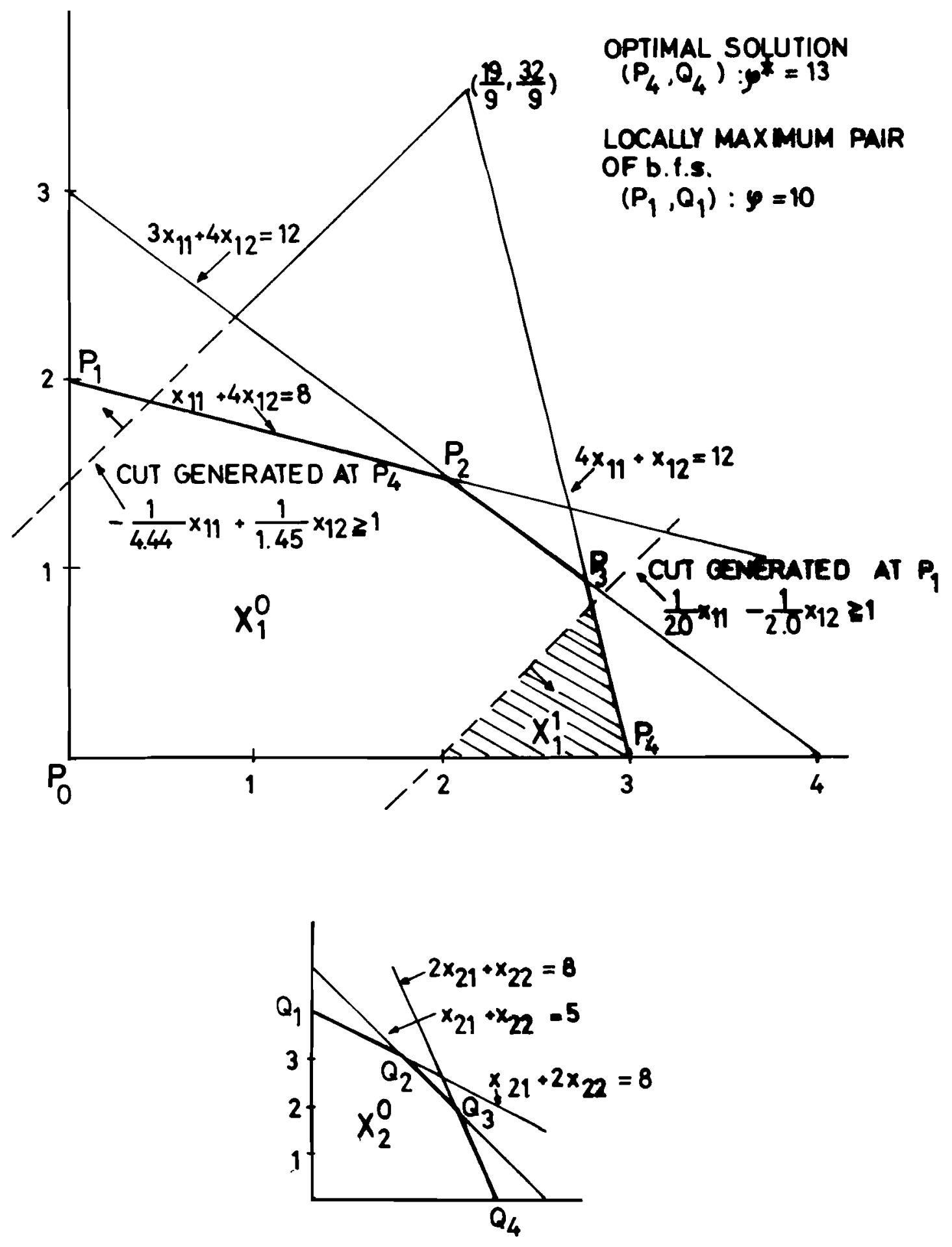

Figure 4.1. A numerical example. 


\begin{tabular}{|c|c|c|c|c|c|}
\hline \multirow[b]{2}{*}{ Problem No. } & \multicolumn{2}{|c|}{ Size of the Problem } & \multirow[b]{2}{*}{$\varepsilon / \phi_{\max }$} & \multirow{2}{*}{$\begin{array}{c}\text { No. of } \\
\text { Local Maxima } \\
\text { Identified }\end{array}$} & \multirow{2}{*}{$\begin{array}{l}\text { CPU time } \\
\text { (sec) }\end{array}$} \\
\hline & $x_{1}$ & $\mathrm{x}_{2}$ & & & \\
\hline 1 & $2 \times 4$ & $2 \times 4$ & 0.0 & 1 & \multirow{4}{*}{$\leq 0.5$} \\
\hline 2 & $3 \times 6$ & $3 \times 6$ & 0.0 & 1 & \\
\hline 3 & $2 \times 5$ & $2 \times 5$ & 0.0 & 1 & \\
\hline 4 & $6 \times 11$ & $6 \times 11$ & 0.0 & 1 & \\
\hline 5 & $3 \times 5$ & $3 \times 5$ & 0.0 & 2 & $\leq 0.5$ \\
\hline 6 & $5 \times 8$ & $5 \times 8$ & 0.0 & 1 & \multirow{3}{*}{1.0} \\
\hline 7 & $3 \times 6$ & $3 \times 6$ & 0.0 & 1 & \\
\hline 8 & $7 \times 11$ & $7 \times 11$ & 0.0 & 1 & \\
\hline 9 & $5 \times 8$ & $5 \times 8$ & 0.0 & 2 & \multirow[t]{2}{*}{0.6} \\
\hline 10 & $9 \times 19$ & $9 \times 19$ & 0.0 & 2 & \\
\hline 11 & $6 \times 12$ & $6 \times 12$ & 0.05 & 5 & \multirow{3}{*}{8.1} \\
\hline 12 & $6 \times 12$ & $6 \times 12$ & 0.01 & 6 & \\
\hline 13 & $6 \times 12$ & $6 \times 12$ & 0.0 & 6 & \\
\hline 14 & $10 \times 22$ & $13 \times 24$ & 0.05 & 3 & 20.7 \\
\hline
\end{tabular}

Problem 2 is taken from [20] and problem 9 from [2].

$11 \sim 13$ are the same problems having six global maxima with equal value. These are in fact global optima. The data for this problem is given below:

$$
c_{1}=0, c_{2}=0, b_{1}=b_{2}=(21,21,21,21,21,21)^{t}
$$




$$
\mathrm{C}=\left[\begin{array}{cccccc}
2 & -1 & 0 & 0 & 0 & 0 \\
-1 & 2 & -1 & 0 & 0 & 0 \\
0 & -1 & 2 & -1 & 0 & 0 \\
0 & 0 & -1 & 2 & -1 & 0 \\
0 & 0 & 0 & -1 & 2 & -1 \\
0 & 0 & 0 & 0 & -1 & 2
\end{array}\right] \quad A_{1}=A_{2}=\left[\begin{array}{cccccc:cccccc}
1 & 2 & 3 & 4 & 5 & 6 & 1 & 0 & 0 & 0 & 0 & 0 \\
2 & 3 & 4 & 5 & 6 & 1 & 0 & 1 & 0 & 0 & 0 & 0 \\
3 & 4 & 5 & 6 & 1 & 2 & 0 & 0 & 1 & 0 & 0 & 0 \\
4 & 5 & 6 & 1 & 2 & 3 & 0 & 0 & 0 & 1 & 0 & 0 \\
5 & 6 & 1 & 2 & 3 & 4 & 0 & 0 & 0 & 0 & 1 & 0 \\
6 & 1 & 2 & 3 & 4 & 5 & 0 & 0 & 0 & 0 & 0 & 1
\end{array}\right]
$$

This is the problem associated with the convex maximization problem

$$
\max \left\{\frac{1}{2} x^{t} C x \mid A_{0} x \leq b, x \geq 0\right\}
$$

Data for problem 14 was generated randomly. 
$\underline{\text { References }}$

[1] Altman, M. "Bilinear Programming," Bullentin de l'Académie Polonaise des Sciences, 16, No. 9(1968), 741-746.

[2] Balas, E. and Burdet, C.-A. "Maximizing a Convex Quadratic Function Subject to Linear Constraints," Management Science Research Report No. 299, GSIA, Carnegie-Melion University, Pittsburgh, Pennsylvania, July 1973.

[3] Cabot, A.V. and Francis, R.L. "Solving Certain Nonconvex Quadratic Minimization Problems by Ranking Extreme Points," Operations Research, 18, No. 1 (1970), 82-86.

[4] Charnes, A. and Cooper, W.W. "Nonlinear Power of Adjacent Extreme Point Methods in Linear Programming," Econometrica, 25 (1957), 132-153.

[5] Candler, W. and Townsley, R.J. "The Maximization of a Quadratic Function of Variables Subject to Linear Inequalities," Management Science, 10, No. 3 (1964), 515-523.

[6] Cottle, R.W. and Mylander, W.C. "Ritter's Cutting Plane Method for Nonconvex Quadratic Programming," in Integer and Nonlinear Programming (J. Abadie, ed.), North Holland, Ams terdam, 1970.

[7] Dantzig, G.B. "Reduction of a 0-1 Integer Program to a Bilinear Separable Program and to a Standard Complementary Problem," Unpublished Note, July 27, 1971.

[8] Dantzig, G.B. "Solving Two-Move Games with Perfect Information," RAND Report P-1459, Santa Monica, California, 1958 .

[9] Falk, J. "A Linear Max-Min Problem," Serial T-251, The George Washington University, Washington, D.C., June 1971 .

[10] Gallo, G. and Ülkücü, A. "Bilinear Programming: An Exact Algorithm," Paper presented at the 8th International Symposium on Mathematical Programming, Stanford University, Stanford, California, August 1973.

[11] Konno, K. "Maximization of Convex Quadratic Function under Linear Constraints," Research Memorandum, International Institute for Applied Systems Analysis, Laxenburg, Austria, 1974. (Forthcoming in Mathematical Programming.) 
[12] Konno, H. "Bilinear Programming Part II: Applications of Bilinear Programming," Technical Report No. 71-10, Department of Operations Research, Stanford University, Stanford, California, August 1971.

[13] Mangasarian, O.L. "Equilibrium Points of Bimatrix Games," SIAM Journal of Applied Mathematics, 12, No. 4 (1964), 778-780.

[14] Mangasarian, O.L. and Stone, H. "Two-Person Nonzero-Sum Games and Quadratic Programming," Journal of Mathematical Analysis and Applications, 9 (1964), 348-355.

[15] Mills, H. "Equilibrium Points in Finite Games," SIAM Journal of Applied Mathematics, $\underline{8}$, No. 2 (19人0), 397-402.

[16] Mylander, W.C. "Nonconvex Quadratic Programming by a Modification of Lemke's Method," RAC-TP-414, Research Analysis Corporation, McLean, Virginia, 1971.

[17] Ritter, K. "A Method for Solving Maximum Problems with a Nonconcave Quadratic Objective Function," $\mathrm{z}$. Wahrscheinlichkeitstheorie, verv. Geb., 4 (1966), 34 0 -351.

[18] Raghavachari, M. "On Connections Between Zero-One Integer Programming and Concave Programming under Linear Constraints," Operations Research, 17, No. 4 (1969), $680-684$.

[19] Tui, H. "Concave Programming under Linear Constraints," Soviet Mathematics (1964), 1537-1440.

[20] Zwart, P. "Nonlinear Programming: Counterexamples to Two Global Optimization Algorithms," Operations Research, 21, No. 6 (1973), 1260-12.

[21] Zwart, P. "Computational Aspects of the Use of Cutting Planes in Global Optimization," in Proceedings of the 1971 Annual Conference of the ACM (1971), 457-465. 